\title{
Multiwavelength analysis of a solar quiet region
}

\author{
G. Tsiropoula ${ }^{1}$, K. Tziotziou ${ }^{1}$, P. Schwartz ${ }^{2}$, and P. Heinzel ${ }^{2}$ \\ 1 National Observatory of Athens, Institute for Space Applications and Remote Sensing, 15236 Palea Penteli, Greece \\ e-mail: [georgia; kostas] @space.noa.gr \\ 2 Astronomical Institute, Academy of Sciences of the Czech Republic, 25165 Ondřejov, Czech Republic \\ e-mail: [schwartz; pheinzel]@asu.cas.cz
}

Received 5 May 2008 / Accepted 10 October 2008

\begin{abstract}
Context. We examine oscillatory phenomena in a solar network region from multi-wavelength observations obtained by the groundbased Dutch Open Telescope (DOT) and by the Coronal Diagnostic Spectrometer (CDS) on the spacecraft Solar and Heliospheric Observatory (SoHO). The observations were obtained during a coordinated observing campaign in October 2005.

Aims. We investigate the temporal variations of the intensities and the velocities in two distinct regions of the quiet Sun, one containing several dark mottles and the other several bright points defining the network boundaries (NB). The aim is to find similarities and/or differences in the oscillatory phenomena observed in these two regions and in different spectral lines formed from the chromosphere to the transition region, as well as the propagation characteristics of waves.

Methods. Intensity and velocity variations are studied with wavelet and phase difference analyses.

Results. Both regions (i.e. mottles and NB) show a periodicity of $\sim 5 \mathrm{~min}$ in all considered lines. The $\mathrm{V}-\mathrm{V}$ phase differences in the NB region point to an upward propagation of waves; in the region of mottles, for periods of 250-400 s, the phase difference is mainly negative, which suggests a downward propagation, in turn indicating a refraction of waves from the inclined magnetic field of mottles along the line-of-sight.

Conclusions. The phase differences at the NB arise from a predominance of upward propagating waves. In the mottles' region, the negative phase differences we found suggest that propagating waves encounter a boundary and are refracted and reflected. Of course, several limitations exist in the exact interpretation of the phase differences, e.g. the complex topology of the magnetic field, the formation conditions and heights of the examined spectral lines, and the low spatial resolution.
\end{abstract}

Key words. Sun: chromosphere - Sun: transition region - Sun: oscillations

\section{Introduction}

It is now well established that the solar photosphere is permeated with strong magnetic fields in the form of flux tubes, which primarily occur at the boundaries of the supergranular cells. The magnetic fields are swept there by the supergranular flow, which results in magnetic flux coalescing at cell vertices with replenishment of flux occurring on a scale of a few days to a week. Higher up, in the chromosphere, the quiet Sun displays a distinct network appearance identical to the supergranular cell structure. In the $\mathrm{H} \alpha$ line, especially in its wings, several elongated dark structures, called mottles, outline the cell boundaries. Even higher up, in the transition region, the network stands out as more stable cellular patterning with bright patches identifying the network boundaries (NB) and enclosing dark areas that correspond to the internetwork (IN). The spatial dichotomy between the NB and the IN is also apparent in the power spectra leading to the suggestion that different physical mechanisms may dominate in each region. The general conclusion is that NB regions show no power of particular interest below $\sim 5 \mathrm{~min}$, in contrast to IN regions that display enhanced power at $\sim 3 \mathrm{~min}$, especially in Dopplershifts.

Different results obtained from different data sets have been reported so far, which sometimes contradict the aforementioned general conclusions. Several authors have studied properties of spectral lines observed in the visible, UV, and EUV parts of the solar spectrum and report that the chromospheric plasma localized in NB oscillates with a dominant period of $\sim 300 \mathrm{~s}$ (Lites et al. 1993; Curdt \& Heinzel 1998; Krijger et al. 2001). Cauzzi et al. (2000) find from the power spectrum computed for the intensity of the $\mathrm{H} \alpha$ line centre that, for both the NB and IN, the power distribution peaks at $\sim 300 \mathrm{~s}$ with no enhanced power detectable in the 3 min range in the IN. This is consistent with observations in the Ca II H by Lites et al. (1993), showing a power peak in the 3 min range only for the velocity fluctuations. Tziotziou et al. (2004) from a wavelet analysis of $\mathrm{H} \alpha$ observations found intensity and velocity periodicities in dark mottles outlining the NB, and thus overlying the IN, in the range $270-450 \mathrm{~s}$, although a $180 \mathrm{~s}$ period is also apparent. Hansteen et al. (2000) have revealed that Doppler shifts in transition region spectral lines in the IN show strong power for periodicities in the range of $160 \mathrm{~s}$ to $250 \mathrm{~s}$ and that relevant oscillations can extend up to periods of $\sim 500 \mathrm{~s}$. They find that the NB emission and velocity are dominated by periodic variations of $\sim 5 \mathrm{~min}$, although a 3 min signal is obvious in the NB emission, and that the signal in the intensity variations is less obvious than in the velocity ones. They also report the intermittent nature of the oscillations with a typical lifetime of 10 to $15 \mathrm{~min}$. Banerjee et al. (2001) point out that intensities of spectral lines formed from the low chromosphere to the transition region show periodic variations with significant power in the range $250 \mathrm{~s}$ to $500 \mathrm{~s}$ with the strongest peaks around $280 \mathrm{~s}$ and $420 \mathrm{~s}$, whereas the Doppler shifts do not show any significant power within this interval, but oscillate with periods close to 660 s. Gömöry et al. (2006) analysing SoHO/CDS 
observations revealed oscillations with a periodicity of $\sim 300 \mathrm{~s}$ in the intensity of the He I line formed in the upper chromosphere. For the same line they found Doppler shift oscillations with periodicities of $220 \mathrm{~s}$ and $450 \mathrm{~s}$, while for the $\mathrm{OV}$ line they found intensity periodicities in the 250-450 s range and Doppler shift periodicities around $250 \mathrm{~s}$ and $450 \mathrm{~s}$. McIntosh et al. (2001) mention, on the basis of a comparison between different data sets, that a mode mixing could occur in the chromosphere, which could explain different results from different data sets.

The mode of the observed waves, whether standing or propagating, the dominant restoring force (gravity, magnetic, gas pressure, etc.) and the resulting propagation through the atmosphere have been the subject of much deliberation. There are two rising difficulties: a) the modelling of the formation of the spectral lines, from which the physical parameters are obtained, is a difficult task, and b) the presence and topology of the magnetic field is complex and dynamic.

Several attempts have been made to describe these oscillations theoretically and numerically. Rosenthal et al. (2002) included MHD effects in their 2D simulation of waves in a magnetized atmosphere and they found that in regions, where the field is significantly inclined to the vertical, the effect is to reflect all, or almost all, of the wave energy back downwards, with the altitude of the reflection being highly variable. In contrast, in the regions where the field is close to vertical or even vertical, the waves that present the characteristics of a pure acoustic oscillation continue to propagate upward, channelled along the magnetic field lines. Khomenko et al. (2008a,b) performed nonlinear 2D numerical simulations of waves inside a flux tube and found different wave patterns depending on the period and the type of the driver.

In this work we present simultaneous observations of a solar network region obtained by space-borne and ground-based instruments during a coordinated campaign as part of Joint Observing Programme 183. The dataset used is described and a multiwavelength analysis is performed through wavelet and global phase difference analyses, for the fluctuations observed in this region to search for wave signatures at different heights of the solar atmosphere and their propagation characteristics.

\section{Observations and data reduction}

In October 2005 we ran a 12-day observational campaign. The aim of that campaign was to collect multi-wavelength observations both from the ground and space that could be used to study the dynamical behaviour of mottles/spicules and other fine structures, observed in different layers of the solar atmosphere. The data analysed in this work were obtained on October 14 and consist of time sequences of cotemporal observations of a quiet region found at the solar disc centre and recorded by the Coronal Diagnostic Spectrometer (CDS) and the Michelson Doppler Imager (MDI) onboard the Solar and Heliospheric Observatory (SoHO), and by the Dutch Open Telescope (DOT) and the Transition Region and Coronal Explorer (TRACE) (Figs. 1 and 2).

\subsection{DOT observations}

Image sequences recorded by the DOT were obtained between 10:15:43-10:30:42 UT. Bursts of 100 frames each were taken at a rate of 6 frames $\mathrm{s}^{-1}$ (exposure time $\leqslant 20 \mathrm{~ms}$ ). The individual frames were carefully aligned and speckle-reconstructed yielding one reconstructed image per burst. The obtained time
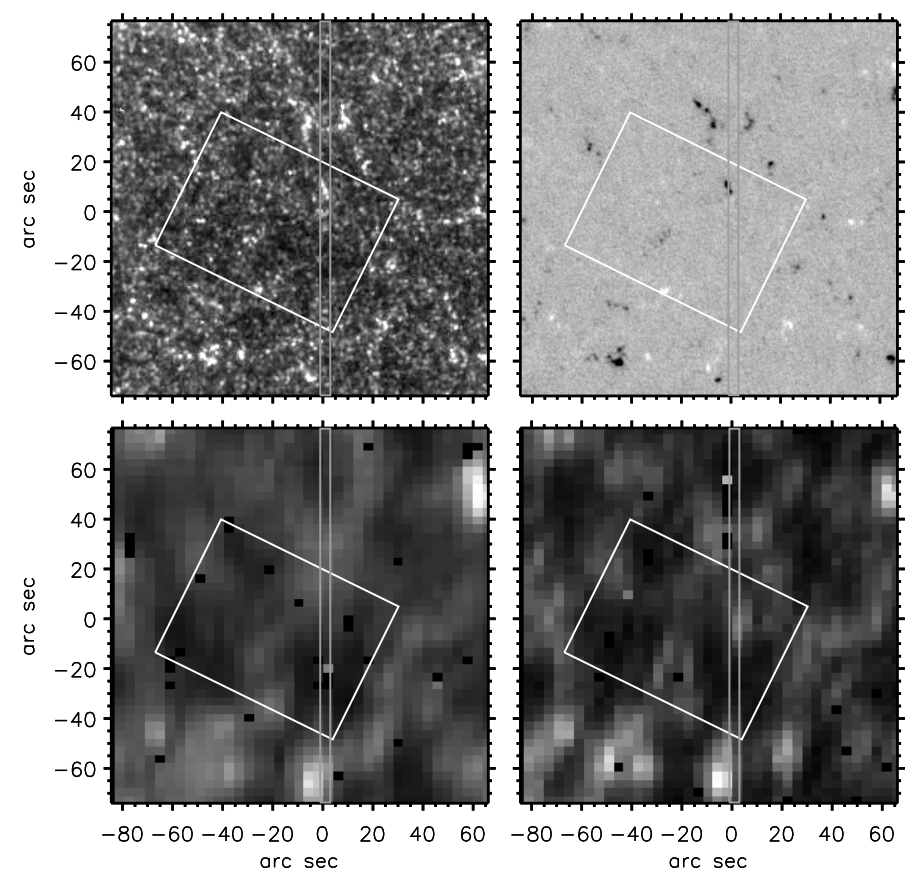

Fig. 1. First row: CIV TRACE image (left) and MDI magnetogram (right) obtained at 10:15 UT. Second row: cut-outs of the CDS raster observations of intensities at $\mathrm{He} \mathrm{I}(l e f t)$ and $\mathrm{O} \mathrm{V}(r i g h t)$ lines obtained from 10:46 to 11:17 UT and corrected for solar rotation due to the time difference. Solar north is up. The white rectangle inside the images marks the DOT's FOV (pointing to the celestial north), which is shown in detail in Fig. 2, while the two parallel grey lines inside the images mark the location of the sit-and-stare CDS observations.

sequence consists of 26 speckle-reconstructed images taken simultaneously at a cadence of $35 \mathrm{~s}$ with a pixel size of $0.071^{\prime \prime}$ in 5 wavelengths along the $\mathrm{H} \alpha$ line profile (i.e. at $-0.7 \AA$, $-0.35 \AA$, line centre, $0.35 \AA$ and $0.7 \AA$ ), in the G-band, in the $\mathrm{Ca}$ II $\mathrm{H}$ line, and in the blue and red continua. The images were carefully aligned and clipped to a common field-of-view (henceforth "FOV") of $78.5^{\prime \prime} \times 59.5^{\prime \prime}$. Details on the telescope, its tomographic multi-wavelength imaging, image aquisition, the speckle reconstruction, and standard reduction procedures are given in Rutten et al. (2004). Figure 2 shows sample images from the whole FOV of the DOT observations in two wavelengths at the wings of the $\mathrm{H} \alpha$ profile.

\subsection{CDS observations}

The CDS data were obtained with the Normal Incidence Spectrometer (NIS) (Harrison et al. 1995). The spectral ranges of NIS ( 310 to $380 \AA$ and 517 to $633 \AA$ ) include lines that are formed over a wide temperature range (approximately $10^{4}$ to $2 \times 10^{6} \mathrm{~K}$ ). On October 14,2005 , the CDS/NIS datasets consist of:

\section{- sit-and-stare observations obtained from 6:44 to 10:46 UT with an effective pixel size of 4 " in the horizontal direction and $3.36^{\prime \prime}$ in the vertical direction with 60 exposures, $40 \mathrm{~s}$ exposure time and cadence of $49 \mathrm{~s}$. Five $49 \mathrm{~min}$ long time series of slit images were obtained; and \\ - six $154^{\prime \prime} \times 240^{\prime \prime}$ raster scans (each one having a duration of $31 \mathrm{~min}$ ) from 10:46 to 13:52 UT and with the same pixel sizes as in the sit-and-stare observations.}

Observations in ten spectral lines spanning the upper solar atmosphere were performed. Table 1 gives the details of the CDS/NIS 

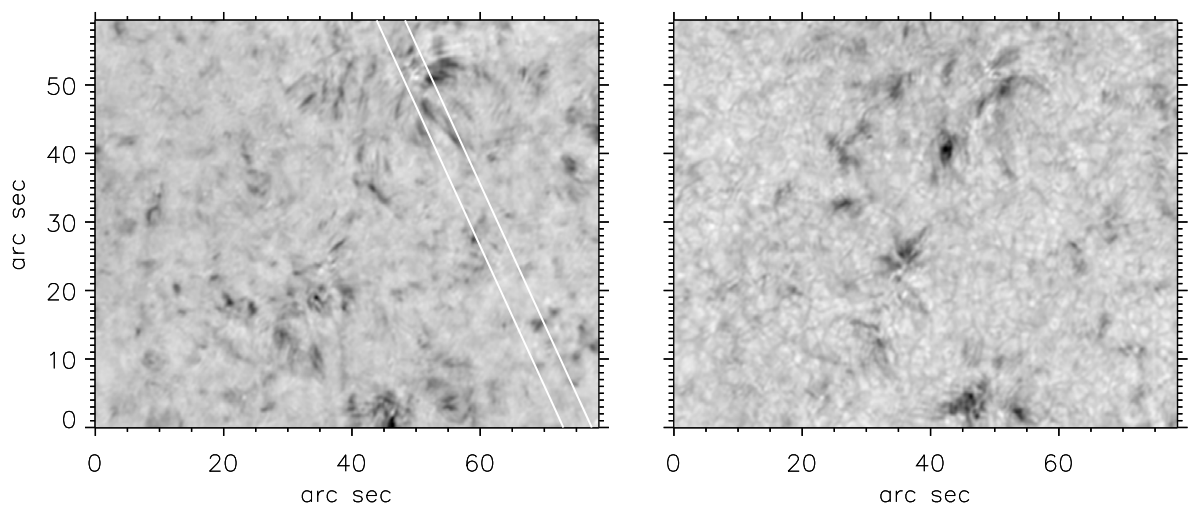

Fig. 2. Common FOV of the observations obtained with DOT and CDS. Left: $\mathrm{H} \alpha-0.7 \AA$, right: $\mathrm{H} \alpha+0.7 \AA$. In the left image the two parallel lines mark the location of the slit during the CDS sit-and-stare observations, which crosses a rosette region in the upper part of the image.
Table 1. The CDS/NIS observations.

\begin{tabular}{lcc}
\hline \hline & Sit-and-stare & Raster \\
\hline slit size & $4^{\prime \prime} \times 240^{\prime \prime}$ & $4^{\prime \prime} \times 240^{\prime \prime}$ \\
XCEN, YCEN & $0.97^{\prime \prime},-0.96^{\prime \prime}$ & $0.305^{\prime \prime},-0.301^{\prime \prime}$ \\
Solar $X$ pixel size & $4^{\prime \prime}$ & $4^{\prime \prime}$ \\
Solar $Y$ pixel size & $3.36^{\prime \prime}$ & $3.36^{\prime \prime}$ \\
FOV & $4^{\prime \prime} \times 240^{\prime \prime}$ & $154^{\prime \prime} \times 240^{\prime \prime}$ \\
No. of rasters & & 6 \\
exposure time & $40 \mathrm{~s}$ & \\
Number of exposures & 60 & \\
Start times & $6: 44,7: 32,8: 21$ & $10: 46,11: 17,11: 48$ \\
of observations & $9: 09,9: 58$ & $12: 19,12: 50,13: 21$ \\
\hline
\end{tabular}

observations. Only the CDS sit-and-stare observations obtained between 9:58 and 10:46 UT, which have a cotemporal part with the DOT observations, have been used in the present study.

Using the standard CDS software the raw measurements were corrected for CCD readout bias, cosmic ray hits, and flat field. In addition to these, a correction was also applied to account for spectrum rotation and line tilt on the CCD. As is known, the plane of dispersion of the NIS is not parallel to the rows of the CCD. Furthermore, there is a misalignment of the entrance slit with the ruling of the normal incidence gratings. Thus individual spectral lines are not perpendicular to the dispersion direction (Brooks \& Bewsher 2006). As shown by Bewsher et al. (2003), the tilt varies with wavelength and between datasets because of its dependence on the temperature of the instrument. These effects can cause an N-S bias in velocity measurements in the solar-y direction. We used the method of Bewsher et al. (2003) to estimate the total tilt for each observed line. We also used the campaign observations (both rasters and sit-and-stare observations) made between October 13 and October 15. The method of Bewsher et al. (2003) was used with two modifications: a) the broadened Gaussian function was used which is commonly used after the SoHO recovery and b) the errors of the velocity-gradient estimates were included in the fitting. After estimates of the total tilt for each observed spectral line, the profiles were fitted using a broadened Gaussian, and the Doppler velocities were corrected taking the total tilt into account.

The average line profiles for the different lines and for the examined sit-and-stare CDS time sequence were obtained by summing over all spatial pixels. Lines with strong blends, those having similar behaviour, or those too weak to be usable were excluded. Thus only the lines He I, O V, and Ne VI were retained for the present analysis. The temperatures of formation of the different ions (last column) were obtained from the CHIANTI 5.2 database (Dere et al. 1997; Landi et al. 2006). Table 2 shows the observed ions used in this work (first column),
Table 2. List of the lines observed with CDS used in this work, their wavelengths, and their temperatures of formation as derived from CHIANTI.

\begin{tabular}{lccc}
\hline \hline Ion & $\begin{array}{c}\text { CDS wavelength } \\
\text { Central wavelength } \\
\text { (from CHIANTI) }\end{array}$ & $\log \left(T_{\mathrm{e}}\right)$ \\
& $(\AA)$ & $(\AA)$ & \\
\hline $\mathrm{He} \mathrm{I}$ & 584.57 & 584.30 & 4.51 \\
$\mathrm{O}$ V & 629.15 & 629.73 & 5.39 \\
$\mathrm{Ne}$ VI & 562.69 & 562.70 & 5.64 \\
\hline
\end{tabular}

their wavelengths as observed by CDS and used for the derivation of the Doppler shifts (second column), as well as their wavelengths as given in the CHIANTI 5.2 database (third column) and the logarithm of their temperature of formation (fourth column). A single Gaussian with a linear background and Poisson statistics were used for fitting each spectral line profile. The data obtained from the fits consist of intensities (i.e. amplitudes of the Gaussian profiles), Doppler shifts, and Doppler widths per spatial pixel per exposure. The shift has been measured relative to the position of the maximum intensity of the spectral line averaged over the whole slit and the entire time series. Negative shifts (dark on the images) correspond to redshifts. A radiometric calibration was also performed to convert the original photon count rates into $\mathrm{erg}^{2} \mathrm{~cm}^{-4} \mathrm{~s}^{-2} \mathrm{sr}^{-2} \mathrm{~A}^{-2}$. After the fitting, we used bilinear interpolation to fill in missing pixels in the data arrays. Figure 1 (second row) shows cut outs of the CDS raster observations of the intensities in the He I and $\mathrm{O} V$ spectral lines. In Fig. 3 we have plotted "space-time" $2 \mathrm{D}$ images of the three parameters obtained from the fitting of the CDS OV $629 \AA$ line profiles. Bright streaks denote the network, while dark ones the internetwork or network cells. Although a complex structure appears, it seems that stronger blueshifts are found in the bright streaks of the intensity plots. On the other hand, no clear relationship can be obtained between Doppler width "space-time" plots and the intensity or Doppler velocity plots. The square root errors of the fitting of the data were also calculated. Only data determined with uncertainty $\chi^{2}$ below $10 \mathrm{erg}^{2} \mathrm{~cm}^{-4} \mathrm{~s}^{-2} \mathrm{sr}^{-2} \mathrm{~A}^{-2}$ were used.

\subsection{TRACE and MDI observations}

TRACE obtained high-cadence filter images at $1550 \AA$, $1600 \AA$, and $1700 \AA$. We used the technique of Handy et al. (1999) to construct an image in C IV line through linear interpolation of the three bandpasses. The high-resolution MDI magnetograms cover a $10.3^{\prime} \times 10.3^{\prime}$ FOV of $1024 \times 1024$ square pixels, each of $0.605^{\prime \prime} \times 0.605^{\prime \prime}$. The area contained several magnetic bipoles and some predominant unipolar regions. Figure 1 (top left and 

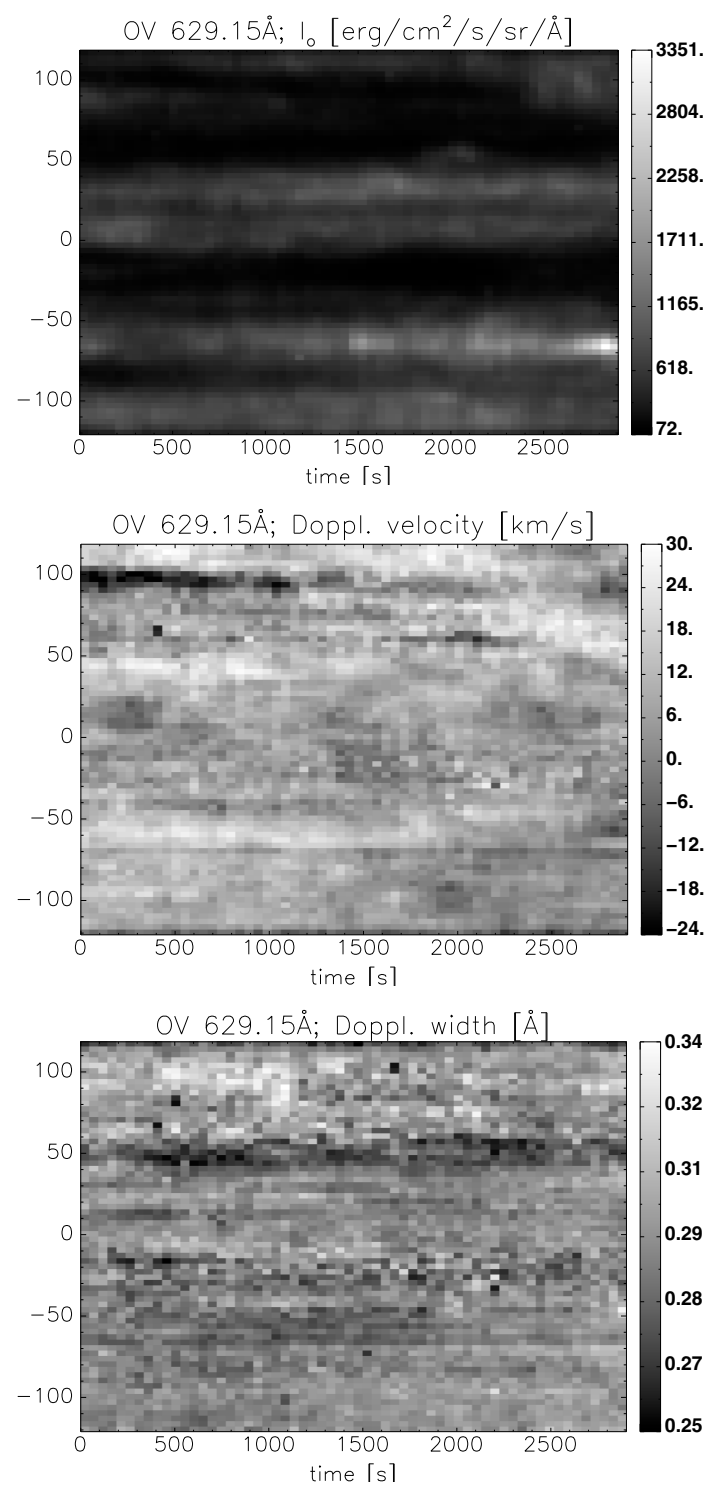

Fig. 3. "Space-time" plots of the three parameters, i.e. peak intensity (top panel), Doppler velocities (middle panel) and Doppler widths (bottom panel) obtained from the fitted profiles of the sit-and-stare CDS observations of the O v $629.73 \AA$ line from 9:58 to 10:46 UT. The abscissa displays the time in seconds, while the ordinate the position along the CDS slit as shown in Fig. 1. Scales are indicated by the vertical bars.

right panels) shows parts of the TRACE C IV image and MDI magnetogram corresponding to the same CDS raster region.

\subsection{Co-alignment of the observations}

Extensive work went into collecting, scaling, and co-aligning the various data sets to a common coordinate system (see Fig. 1 showing TRACE C IV, MDI, DOT, and CDS images co-aligned and scaled to a common coordinate system). Co-alignment between the MDI and TRACE datasets was possible because of the presence of several bright points in the TRACE C IV images that have high magnetic fluxes. Co-alignement between the CDS raster scans and TRACE and MDI images was done by using the XCEN and YCEN values found in the headers of the corresponding images, as well as by the observed network outlines. The CDS sit-and-stare observations were then co-aligned according to the CDS raster scan. The cross-alignment between the
DOT images and the CDS sit-and-stare observations was done by using several bright points visible in the $\mathrm{H} \alpha-0.7 \AA$, Ca II H, G-band, and TRACE C IV, as well as the high MDI magnetic fluxes. In Fig. 1 (first row), part of the FOVs of TRACE C IV image (left panel) and MDI magnetogram (right panel) are shown. In the second row of the same figure, a cut-out of the CDS raster observations of the same region in He I (left panel) and O V (right panel) obtained from 10:46 to 11:17 UT and corrected for solar rotation due to the time difference are shown.

\section{Results}

\subsection{Observations overview}

In the $\mathrm{H} \alpha-0.7 \AA$ DOT image (H $\alpha$ blue wing, Fig. 2 first row, left panel), the dark streaks are part of the elongated $\mathrm{H} \alpha$ mottles seen better at line centre. Some mottle endings appear extra dark in the blue wing image due to Doppler blueshifts. Near the mottle endings, one can see several bright points. They constitute the magnetic network that partially outlines the boundaries of the supergranular cells. A curve fitting of the observed profiles was used to determine the Doppler velocity $v$, which was calculated from the lineshift $\Delta \lambda$ of the $\mathrm{H} \alpha$ line centre through the well known formula $v=c \Delta \lambda / \lambda_{0}$, where $\lambda_{0}$ is the $\mathrm{H} \alpha$ linecentre wavelength and $c$ the speed of light. We then constructed 2D Doppler velocity images for the whole time series.

In CDS sit-and-stare observations, the quiet Sun is dominated by a pattern, which is manifested as a mosaic of bright streaks defining the NB areas and dark streaks defining the IN areas. In Fig. 4 we plotted the variations in the values of the three CDS parameters derived from the fitting of the profiles along the slit for each exposure and for three spectral lines (He I, O V, and Ne VI) and overplotted the temporal average. The high intensity values at $\sim-70^{\prime \prime}$ mark the presence of a blinker, which is also obvious in Fig. 3. We computed the correlation coefficients between the three different parameters. A strong positive correlation $(\sim 0.94)$ is found between the respective peak intensities of the He I line and the $\mathrm{OV}$ and Ne VI lines. These expected strong correlations suggest a strong association between different heights of the solar atmosphere, indicating that we are possibly observing manifestations of the same structural topology at different heights.

In addition, the correlation of the Doppler shifts and Doppler widths are strong between the He I line and the O V line $(0.71$ and 0.80 , respectively), while the corresponding values between the He I and the Ne VI line show very low correlation $(0.36)$ or no correlation at all. Again the strong correlations between the respective Doppler shifts and widths of the He I and $\mathrm{OV}$ lines reflect the aforementioned association between different heights of the atmosphere, while the low correlations between the He I and Ne VI Doppler shifts and widths mainly reflect the presence of a strong dispersion in the derived Ne VI Doppler shifts and widths, which is clearly visible in Fig. 4 . We should remind the reader that the Ne VI line is the noisiest of the three lines (there are substantially fewer observed counts than in the other two lines). A very low correlation is observed between peak intensities and velocities of the same line, for all lines, and an anticorrelation between peak intensities and Doppler widths, as well as between velocities and Doppler widths of the same line, for all lines. However, no useful conclusions can be derived since the exact formation properties of these lines, hence the association between intensity, Doppler shift, and Doppler width, cannot really be known without detailed radiative transfer calculations. 

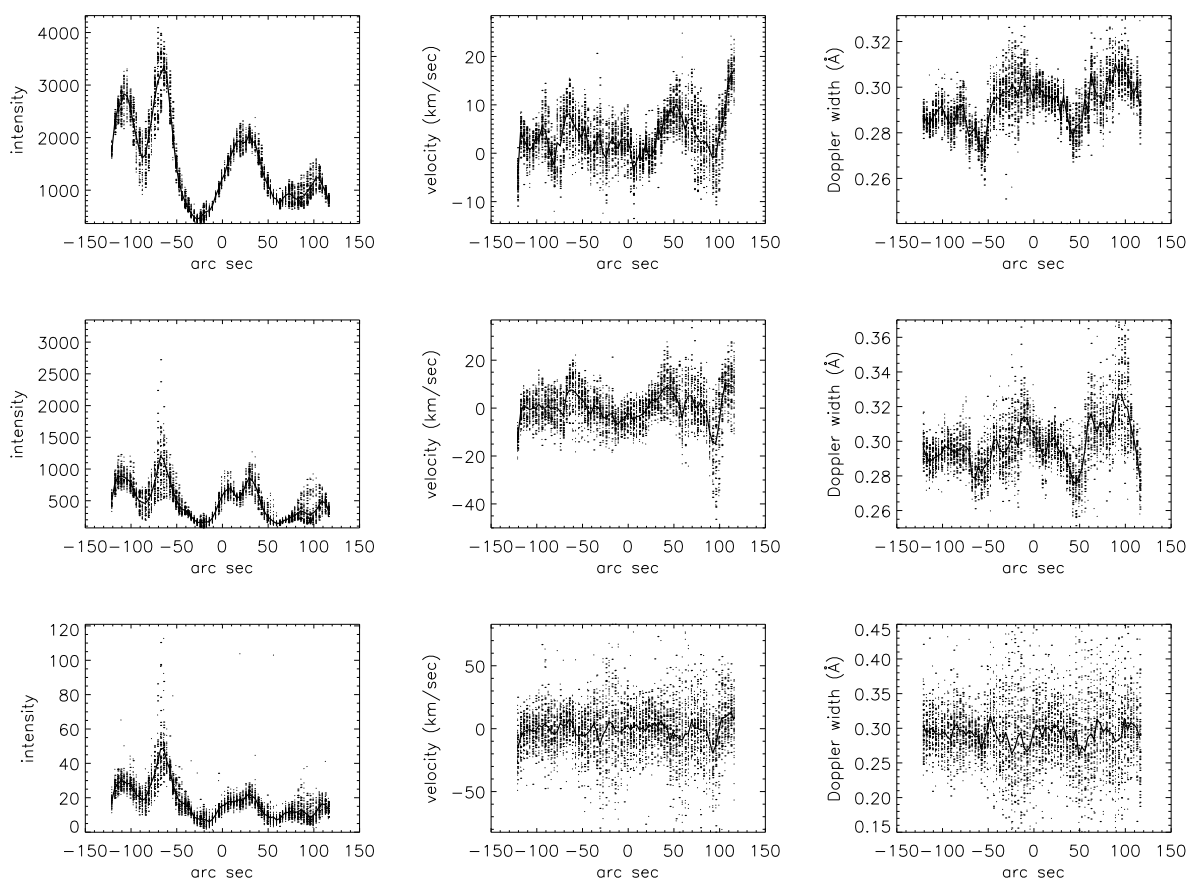

Fig. 4. Temporal variations of the three parameters, i.e. peak intensity (left panel), Doppler velocity (middle panel), and Doppler width (right panel) obtained from the fitted profiles of the CDS sit-and-stare observations for the He I (first row), OV (second row), and Ne VI ((third row) lines from 9:58 to 10:46 UT for each pixel along the slit and each exposure. The overplotted thick line is the temporal average.

\subsection{Intercomparison between sets of observations obtained by different instruments}

Part of the slit (from $-47^{\prime \prime}$ to $19^{\prime \prime}$ ) of the CDS sit-and-stare observations crosses the DOT's FOV and, moreover, from $-10^{\prime \prime}$ to $19^{\prime \prime}$ crosses the rosette region, which is apparent in the upper part of the DOT's FOV and consists of several mottles (see Figs. 3 and 2, left panel).

From the CDS low spatial resolution observations, it is evident that the network boundaries appear as a continuous patch of emission surrounded by dark areas that remain largely unchanged during the $48 \mathrm{~min}$ observation interval. The mottles region appears darker than the NB, but brighter than the IN. The high spatial resolution DOT images, on the other hand, reveal several small bright points, which define the network boundaries and a large number of thin dark structures threading the network and the IN as well. To illustrate the power of high resolution, we rebinned DOT images to CDS spatial pixel sizes both in the $\mathrm{X}$ - and $Y$-direction for intercomparison. The resulting image is shown in Fig. 5. It is obvious that every fine structure disappears after binning. What remains is a bright patch in the place where network bright points were very clearly seen in the DOT images, and a dark patch in the respective region of dark mottles.

\subsection{Wavelet power analysis}

For the study of the temporal variations, we are using a wavelet analysis (Torrence \& Compo 1998) that not only permits the determination of any periodic signal but also shows how this period varies with time. We refer the reader to Tziotziou et al. (2004) for a detailed description of the wavelet analysis method. Periods higher than $600 \mathrm{~s}$ have been filtered out from the time series before the wavelet analysis using an inverse fast Fourier analysis of the time series combined with a high-pass frequency filter, which tends to retain the low-period information while reducing the high-period one. This was done because of a dominant $1000 \mathrm{~s}$ period in the wavelet spectra of the CDS lines and will be further discussed later.

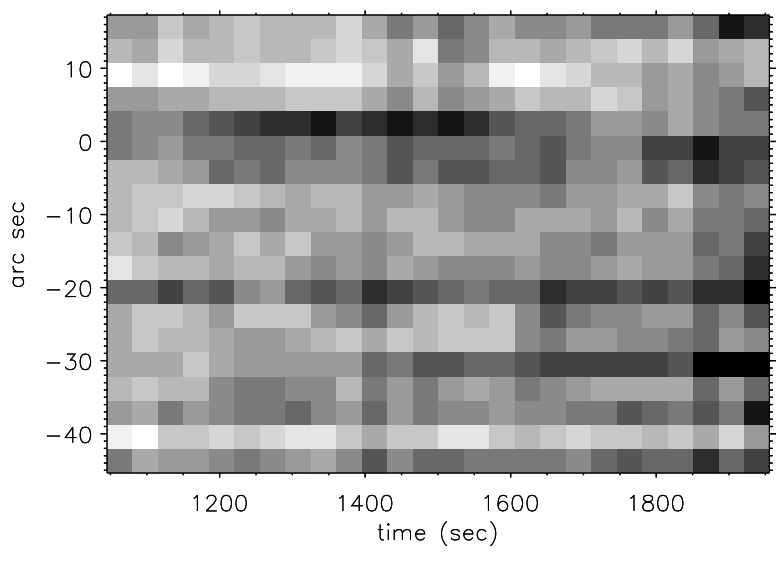

Fig. 5. DOT H $\alpha$ line centre sit-and-stare image after degrading to the CDS spatial resolution. The coordinates correspond to the cospatial and cotemporal observations between DOT and the sit-and-stare CDS as seen in Fig. 2.

We chose two regions: one containing several bright points that define the NB and the other found at the location where several dark mottles are observed changing continuously. These regions correspond to locations from $\sim 2^{\prime \prime}$ to $\sim 8^{\prime \prime}$ and $\sim-7^{\prime \prime}$ to $\sim 0^{\prime \prime}$ along the slit of the CDS and DOT sit-and-stare observations, respectively (see Figs. 3 and 5). We sum over two CDS and DOT pixels to improve the signal-to-noise ratio. We must keep in mind, that due to the low CDS spatial resolution and to the summing, these regions correspond to a cluster of spatially unresolved network bright points in the first case and to several mottles in the second. The region containing the mottles does not correspond to the IN and it is rather brighter than it in all transition region lines as can be seen in Fig. 3. In Figs. 6 to 10 we show examples of the results of the wavelet analysis. In each of these examples, the top panels show the intensity or velocity variations of the unfiltered data (thick solid line, left abscissa) and the filtered data (thin solid line, right abscissa). The middle left panels show the calculated power spectra as a function of time and period for both filtered data (filled grey-scale 

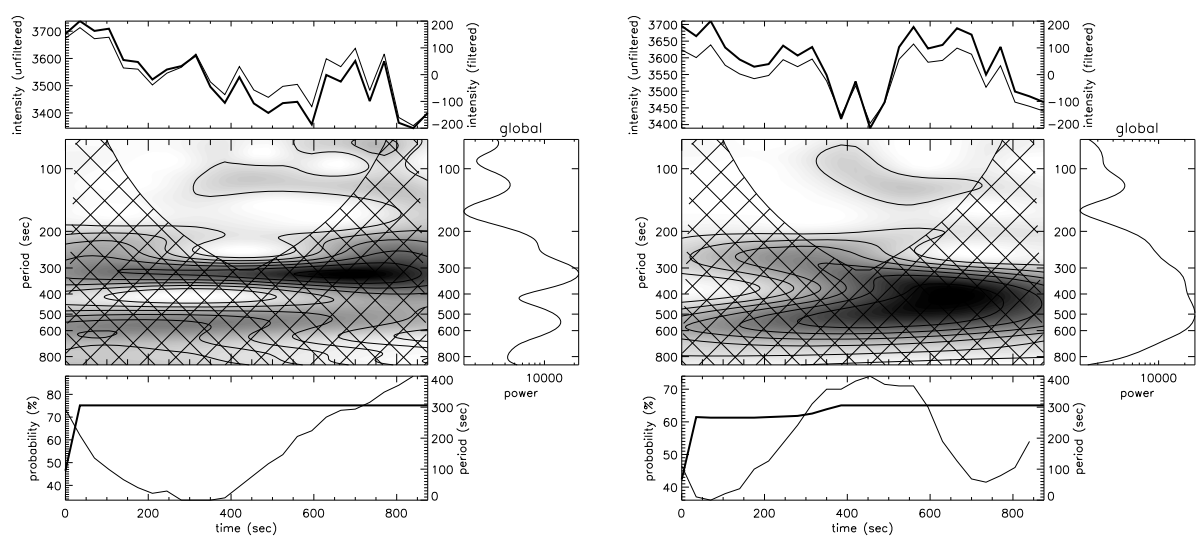

Fig. 6. Wavelet analysis at a fixed location in the mottles' region (left panel) and the $\mathrm{NB}$ region (right panel) for the $\mathrm{H} \alpha$ linecentre intensity variations. For a description of the presented panels see Sect. 3.3.
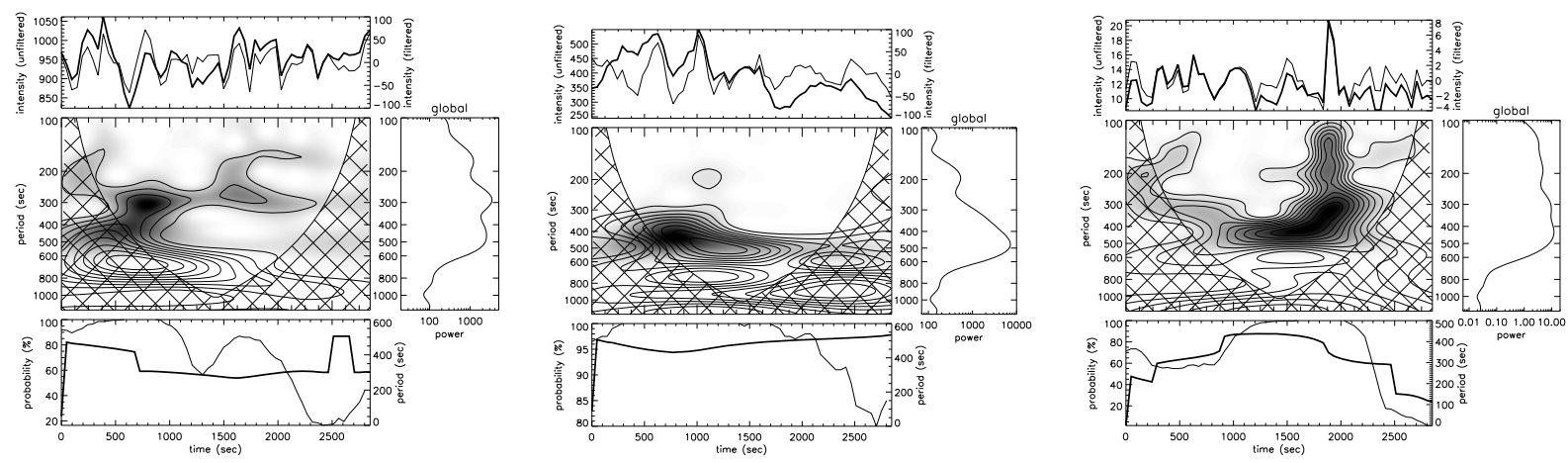

Fig. 7. Wavelet analysis at a fixed location in the mottles' region of the He I (left), O v (centre) and Ne VI (right) peak intensity variations.
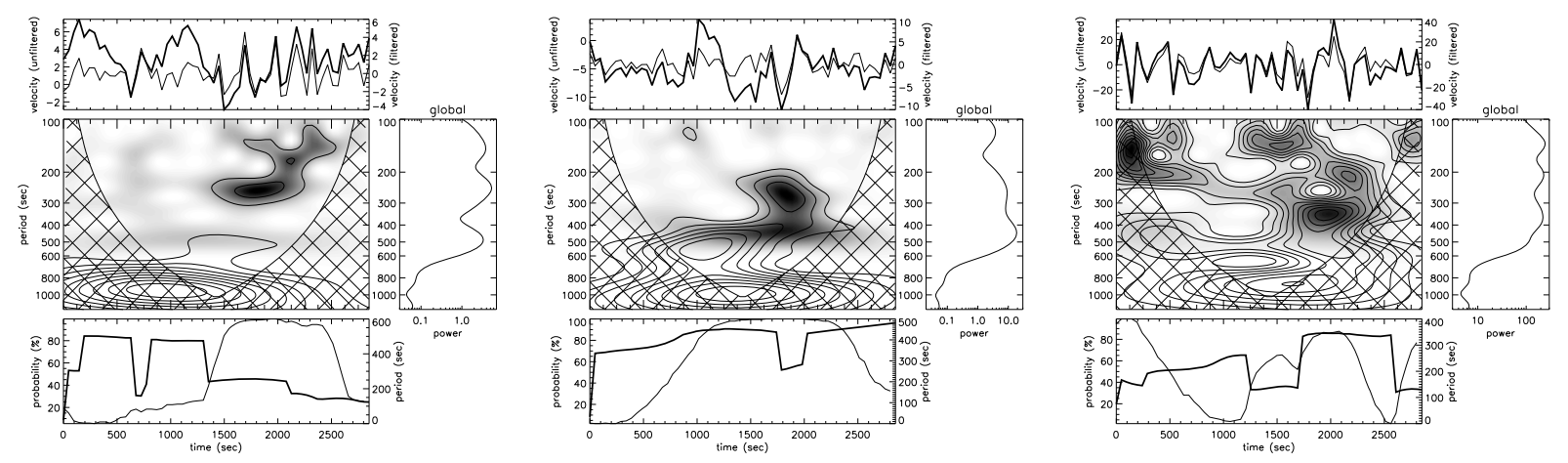

Fig. 8. Wavelet analysis at a fixed location in the mottles' region of the He I (left), O v (centre) and Ne VI (right) velocity variations.

contours) and unfiltered data (line contours). The cross-hatched regions below the cone-of-influence (COI) line indicate the regions that are subject to edge effects due to zero padding. The middle right panels show the global power spectrum for the filtered data, which is the average of the wavelet power spectrum of the filtered data over time. Finally, the lowest panels show the variations in the probability (thin solid line, left abscissa) obtained with the randomization method for the filtered data (see Tziotziou et al. 2004, for details) and the equivalent period of the maximum power peak (thick solid line, right abscissa) for which this probability was calculated. The calculation of the statistical significance of a period by comparison to an assumed distribution of random errors (e.g. Poisson) is obviously constrained by the choice of a specific noise model. However, this randomization test, where the significance is calculated with respect to all possible arrangements of the observed data, is a more reliable, non-parametric approach, since no distributional assumptions of random errors are required.
In Fig. 6 we show the results of the wavelet analysis for the $\mathrm{H} \alpha$ line-centre intensity variations at a fixed location at the mottles' region (left panel) and at the network boundaries (right panel). It is obvious that the used high-pass frequency filtering has hardly any influence on the data. The $\mathrm{H} \alpha$ line-centre intensity variations show a most prominent peak at a period of $\sim 300 \mathrm{~s}$ in the mottle's region and an extended peak at periods found in the range 300-600 s in the NB region. Although the time series is limited, hence a major part of the power of the most dominant periods is subjected to edge effects and lies outside the COI, which is also reflected in the calculated probabilities, its persistence over the whole time series indicates its physical importance. Furthermore, the result regarding the periodicity in the mottle's region is consistent with the results of Tziotziou et al. (2004), who also find similar periodicities in $\mathrm{H} \alpha$ mottles.

The wavelet analysis performed in the He I, O V, and Ne VI CDS observations is shown in Figs. 7 to 10. All spectra of unfiltered data are dominated by a period around $1000 \mathrm{~s}$, which is 

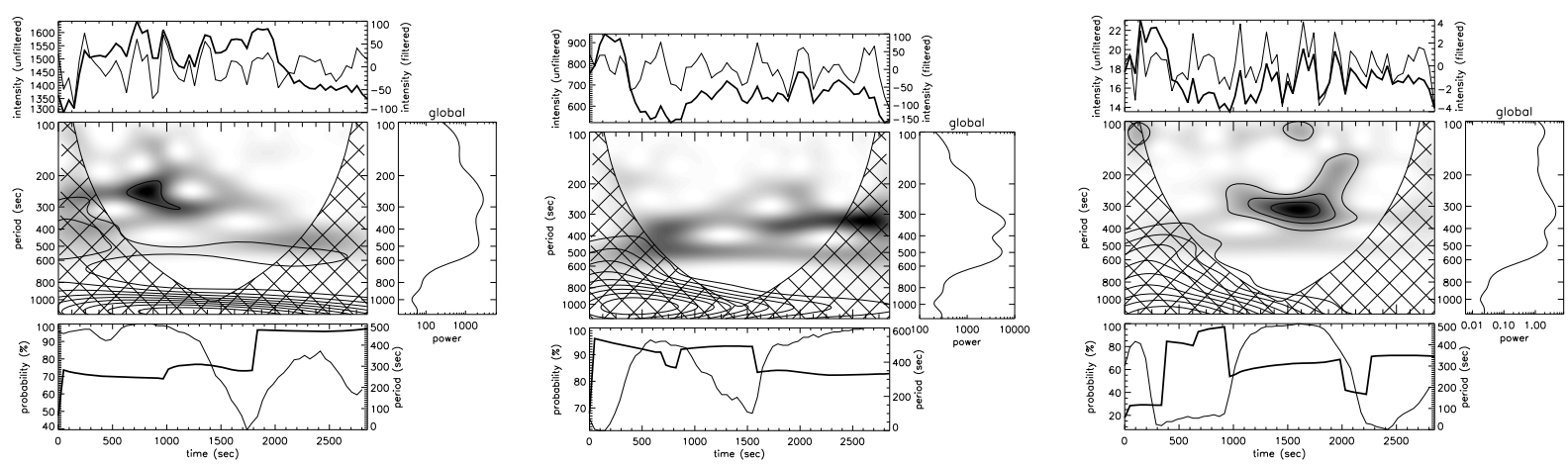

Fig. 9. Wavelet analysis at a fixed location in the NB region of the He I (left), O V (centre) and Ne VI (right) peak intensity variations.
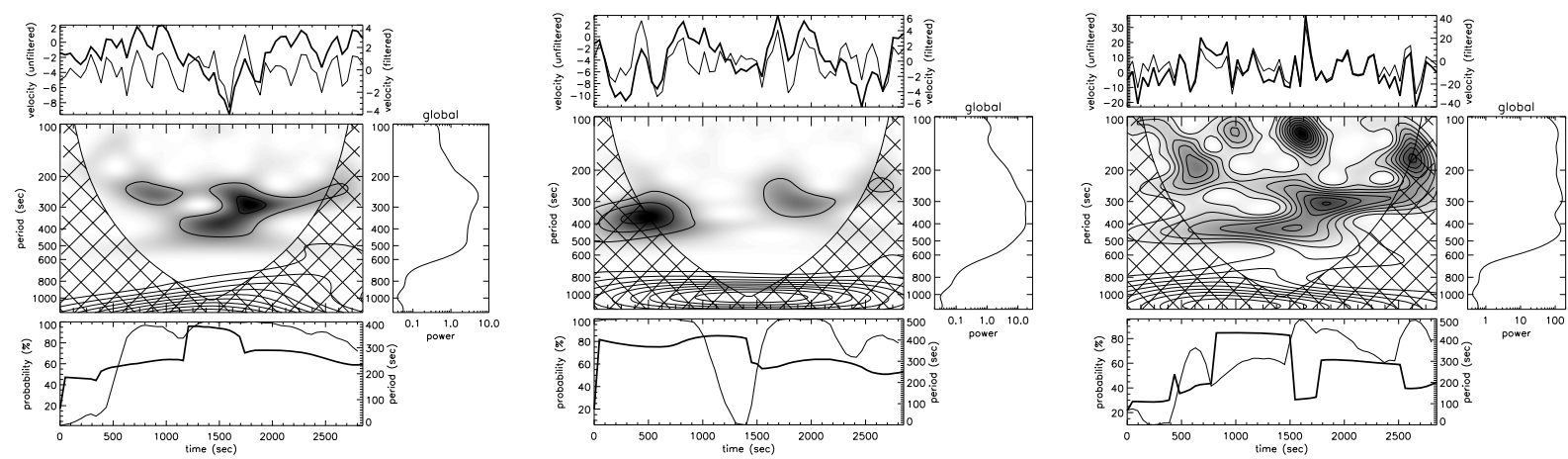

Fig. 10. Wavelet analysis at a fixed location in the NB region of the He I (left), O V (centre) and Ne VI (right) velocity variations.

mostly outside the COI, hence subjected to edge effects. It is unclear whether this period is physically important and associated with the lifetime of mottles. The high-pass frequency filtering, most of the time, has a minimal influence on the data as an inter-comparison of the filtered and unfiltered time series clearly shows. It is obvious that it has simply removed the aforementioned period of $1000 \mathrm{~s}$ as the presented filtered and unfiltered power spectra indicate. Both intensity and velocity filtered power spectra show several periods with significant power within the COI. Most spectra in the mottle's region, as well as in the NB, show dominant oscillation signatures mainly in the $250-400 \mathrm{~s}$ range with rather variable probability ranging mainly from $80 \%$ to $100 \%$.

\subsection{Global phase difference analysis}

To look for propagation characteristics of waves at different heights in the solar atmosphere, we computed the phase difference between the $\mathrm{H} \alpha$ line-centre filtered intensity and velocity time series and the corresponding filtered time series of the He I, $\mathrm{O} v$, and Ne VI lines observed with CDS. To find the phase difference, a cross-wavelet transform is used (see Tziotziou et al. 2005). We should point out that phase difference between unfiltered data show an almost identical behaviour but have not been used for reasons of consistency with the rest of the paper.

In Figs. 11 and 12, we respectively present the global I-I and $\mathrm{V}-\mathrm{V}$ phase differences as functions of period for the filtered time series. No wraparound corrections have been made. Phase difference values are between $\pi$ and $-\pi$ and values just above $\pi$ are transformed into values just below $-\pi$. The I-I phase spectra (Fig. 11) are difficult to interpret, because the I-I phase spectra depend on many parameters, such as frequency, height in the atmosphere, and the peculiarities of the line formation. However, the $\mathrm{V}-\mathrm{V}$ phase difference in Fig. 12 is very suggestive. It is clear from this figure that the waves in the NB (Fig. 12, right) propagate vertically, since above $\sim 200 \mathrm{~s}$ there is always a constant inclination of the phase curve with period for all lines, also taking the wraparound effect into account. This is exactly what is expected from the theoretical behaviour of the vertically propagating waves. At periods above $\sim 350 \mathrm{~s}$, the phase difference becomes almost constant for a large range of periods meaning that there is no propagation for these periods. This is also what is expected and it means that the cut-off period in the NB is about $350-400 \mathrm{~s}$. It is also remarkable that its values for the different line pairs have an almost similar behaviour indicating a clear interconnection between processes in the lower and higher solar atmosphere and in both the mottles' and the NB region. The difference in the inclination of the phase curves for the different spectral lines suggests that they form at slightly different heights, $\mathrm{Ne}$ VI forms the highest and the He I the lowest. The V-V phase differences in the mottles's region are different. There is no constant dependence on the period above $200 \mathrm{~s}$, as in the case of the NB. This means that there is no vertical propagation and the waves measured from the same observational pixel at different heights have nothing to do with each other. They may be waves propagating at different inclined mottles along the line-ofsight. On the other hand, since the calculated phase differences are mostly negative, this suggests that there is actually a downward propagation dominating in this region, at least for periods of $250-400 \mathrm{~s}$. This can be explained as due to waves refracted from the inclined magnetic field of mottles or converted to other wave types, such as fast or slow MHD modes (depending on the field strength).

Comparison between the global phase differences of the He Iformed in the upper chromosphere and the two transition region lines shows almost negligible differences implying 

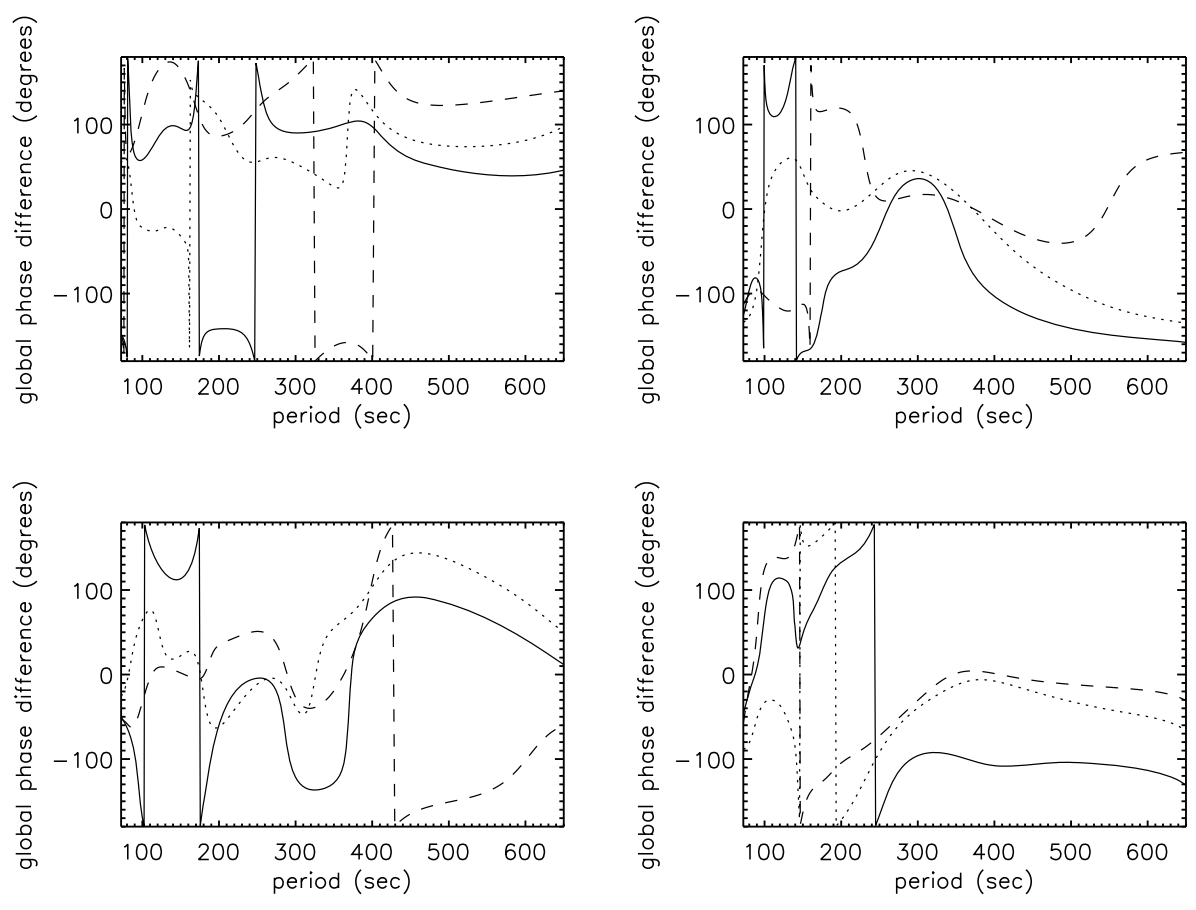

Fig. 11. Left: global phase difference (in degrees) of filtered intensities as a function of period relative to the $\mathrm{H} \alpha$ line-centre intensity (He I (thick solid line), O V (dotted line), Ne VI (dashed line) intensities) for the mottles region. Right: the same for the NB region.

Fig. 12. Left: global phase difference (in degrees) of filtered velocities as a function of period relative to the $\mathrm{H} \alpha$ line-centre Doppler velocity (He I (thick solid line), O v (dotted line), Ne VI (dashed line) velocities) for the mottles' region. Right: the same for the NB region. non-propagative signature of the standing waves in this upper part of the atmosphere in accordance to the aforementioned discussion of the $\mathrm{V}-\mathrm{V}$ spectra between $\mathrm{H} \alpha$ and the three lines.

\section{Conclusions}

In this paper we analysed observations obtained during a coordinated campaign from a ground-based telescope (DOT) and from spacecraft (SoHO). Fortunately, the low spatial resolution $\mathrm{CDS} / \mathrm{SoHO}$ sit-and-stare observations in several transition region lines crossed a rosette region seen in high-resolution DOT observations. The DOT observations in $\mathrm{H} \alpha$ indicate that this region consists of two subregions in which we have concentrated our studies: one containing several bright points, co-spatial with strong magnetic field at the photospheric level, as seen in MDI magnetograms, that define the network boundaries; the other consisting of several dark mottles. Rebinning the DOT pixels to CDS spatial pixel sizes allowed us to show that every fine scale structure disappears, and what remains is a bright patch in the place where network bright points were seen and a relatively dark patch in the place of mottles, also indicating the limitations of the low spatial resolution of the CDS observations for analysing fine structures. This patch persists in the CDS images; however, it is more extended and contains the place where mottles are seen underneath. The region containing the dark mottles is rather bright in the low-resolution CDS images, although less bright than the region containing the bright points (NB). This could indicate the presence of a canopy above mottles or the change in the appearance of these structures as they enter the upper atmospheric layers due to changes of their physical parameters.

A wavelet analysis allowed us to detect intensity and velocity oscillations for a set of lines covering a wide range of temperatures starting from the strong chromospheric $\mathrm{H} \alpha$ line to the upper chromospheric $\mathrm{He} \mathrm{I}$ and transition region $\mathrm{OV}$ and $\mathrm{Ne} \mathrm{VI} \mathrm{lines.} \mathrm{In} \mathrm{the} \mathrm{intensity} \mathrm{and} \mathrm{velocity} \mathrm{power} \mathrm{spectra} \mathrm{of} \mathrm{these}$ lines, there is little difference between the mottles' region and the NB. Both average (global) unfiltered power spectra show a dominant peak around $1000 \mathrm{~s}$, which is subjected to edge effects and it is unclear whether it is physically associated with the lifetime of mottles. However, both average (global) filtered/unfiltered power spectra also show significant peaks in the 5-min range. Peaks in the 3-min range are hardly observed and, when observed, they are not that significant. This is consistent with the general finding of a dominant 5-min periodicity in the NB, and also with the finding of Tziotziou et al. (2004), who found a 5 -min periodicity in intensity and velocity variations of dark mottles observed in $\mathrm{H} \alpha$. This period contrasts to the clear 3-min periodicity of IN regions, which is the chromospheric cut-off period, and is compatible with the idea that leakage of photospheric 5-min acoustic oscillations (p-modes) are important in the formation of mottles (De Pontieu et al. 2004). There is a general consensus in recent years that the acoustic cut-off period depends on the inclination of the magnetic field lines to the vertical (Suematsu 1990; De Pontieu et al. 2004). Thus in mottles formed along inclined magnetic fields, the acoustic cut-off period increases, allowing p-modes to escape into higher layers. We should also point out that measured periods in IN regions of the order of $5 \mathrm{~min}$ reported in the literature (see Introduction) could be because that part of the IN regions close to the NB are covered by inclined mottles, meaning this period could be attributed to their presence.

Propagation characteristics of waves at different heights in the solar atmosphere can be derived from the I-I and $\mathrm{V}-\mathrm{V}$ global phase differences between the chromospheric $\mathrm{H} \alpha$ line and the upper chromospheric He I and transition region lines $\mathrm{OV}$ and Ne VI for both subregions, i.e. mottles and NB. Global phase differences indicate that there is a strong mechanical coupling from the chromosphere to the transition region. The I-I phase spectra are difficult to interpret, due to their dependence on many parameters, such as frequency, formation heights of the lines, and peculiarities in the formation of the different lines. In contrast, the $\mathrm{V}-\mathrm{V}$ phase spectra are very suggestive. In the NB region for periods in the range $200-350 \mathrm{~s}$, the inclination of the phase curves with period points to upward propagating waves. At periods above $\sim 350 \mathrm{~s}$, the phase difference becomes almost constant for a wide range of periods and for all lines, meaning that there is no propagation for these periods. In the 
mottles' region, on the other hand, the $\mathrm{V}-\mathrm{V}$ phase spectra are more difficult to interpret, since there is no such constant dependence on the period, as in the NB case. It may mean that there is no vertical propagation and the waves are propagating at different inclined mottles along the line-of-sight, or it could even indicate the presence of a canopy above the mottles in higher atmospheric layers. There is also a phase difference for periods of 250-400 s that is mainly negative, a finding that suggests that there is actually a downward propagation dominating the mottles' region, indicating a refraction of the waves from the inclined magnetic field of mottles or even conversion to other wave types, such as fast or slow MHD modes (depending on the field strength). In recent years there have been several observational and theoretical findings supporting the view that, when propagating waves encounter a boundary across which there is a change in the dispersive characteristics of the medium, they are refracted and reflected. Numerical simulations have shown that the region where the magnetic and gas pressures become comparable $(\beta \sim 1)$ provides such a boundary (see Bogdan et al. 2003 and references therein). Thus the $\beta$-transition region partitions the atmosphere into contiguous volumes of high- $\beta$ and low- $\beta$ plasma and acts as a magnetic canopy for the waves. In the quiet Sun, this canopy is expected to vary in height between roughly 0.8 and $1.6 \mathrm{Mm}$ above the base of the photosphere, and it would influence the propagation characteristics of the observed waves. However, the exact influence is not known and is highly model dependent.

It should be stressed that several limitations and difficulties appear in the exact interpretation of the phase differences. One problem resides in the presence and the topology of the magnetic field. Its complexity and dynamics might explain the complexity of the obtained phase differences. Another problem resides in the formation of the $\mathrm{H} \alpha$ and $\mathrm{He}$ I lines, which are both formed under non-LTE conditions. Furthermore, we should point out that, in this work, the analysis of the DOT data was made by averaging over $4^{\prime \prime}$ in the $X$-direction and $3.36^{\prime \prime}$ in the $Y$-direction in order to match the resolution of the CDS data. We would thus expect that we have averaged over several waves of different phases. All the above difficulties and limitations could explain the wide range of different and sometimes contradictory results about the periods of waves and their propagation characteristics in quiet Sun network regions found in the literature (see Introduction) and why their theoretical understanding remains uncomplete.
Despite these difficulties, our analysis strongly suggests that the phase differences at the NB arise from a predominance of upward propagating, most probably, acoustic waves. A correct and exact interpretation would, however, require simulations, due to the increasing importance with height of nonlinear, nonadiabatic wave motions, and the lack of LTE and ionization equilibrium in the formation regions of the lines.

Acknowledgements. This work has been partly supported by a Greek-Czech programme of cooperation. P.S. and P.H. are also supported by the ESAPECS project No 98030. The DOT is operated by Utrecht University at the Spanish Observatorio del Roque de los Muchachos of the Instituto de Astrofísica de Canarias. MDI and CDS is part of SoHO, the Solar and Heliospheric Observatory, which is a mission of international cooperation between ESA and NASA. CHIANTI is a collaborative project involving the NRL (USA), RAL (UK), and the following Universities: College London (UK), Cambridge (UK), George Mason (USA), and Florence (Italy). We would like to thank Elena Khomenko for very useful discussions concerning the intrepretation of phase differences and waves. We are grateful to the SoHO and TRACE science planning teams, especially to P. Young, U. Schühle, S. Gregory, and K. Schrijver.

\section{References}

Banerjee, D., O’Shea, E., Doyle, J. G., \& Goossens, M. 2001, A\&A, 371, 1137 Bewsher, D., Parnell, C. E., Pike, C. D., \& Harrison, R. A. 2003, Sol. Phys., 215, 217

Bogdan, T. J., Carlsson, M., Hansteen, V. H., et al. 2003, ApJ, 599, 626

Brooks, D. H., \& Bewsher, D. 2006, Solar Phys., 234, 257

Cauzzi, G., Falchi, A., \& Falciani, R. 2000, A\&A, 357, 1093

Curdt, W., \& Heinzel, P. 1998, ApJ, 503, L95

De Pontieu, B., Erdélyi, R., \& James, S. P. 2004, Nature, 430, 536

Dere, K. P., Landi, E., Mason, H. E., et al. 1997, A\&AS, 125, 149

Gömöry, P., Rybák, J., Kučera, A., Curdt, W., \& Wöhl, H. 2006, A\&A, 448, 1169

Handy, B. N., Acton, L. W., Kankelborg, C. C., et al. 1999, Sol. Phys., 187, 229

Hansteen, V. H., Betta, R., \& Carlsson, M. 2000, A\&A, 360, 742

Harrison, A., Sawyer, E . C., Carter, M. K., et al. 1995, Sol. Phys., 162, 233

Khomenko, E., Collados, M., \& Felipe, T. 2008a, Sol. Phys., 251, 589

Khomenko, E., Centeno, R., Collados, M., \& Trujillo Bueno, J. 2008b, ApJ, 676, L85

Krijger, J. M., Rutten, R. J., Lites, B. W., et al. 2001, A\&A, 379, 1052

Landi, E., Del Zanna, G., Young, P. R., et al. 2006, ApJ, 162, 261

Lites, B. W., Rutten, R. J. \& Kalkofen, W. 1993, ApJ, 414, 345

McIntosh, S. W., Bogdan, T. J., Cally, P. S., et al. 2001, ApJ, 548, L237

Rosenthal, C. S., Bogdan, T. J., Carlsson, M., et al. 2002 ApJ, 564, 508

Rutten, R. J., Hammerschlag, R. H., Bettonvil, F. C. M., Sütterlin, P., \& de Wijn, A. G. 2004, A\&A, 413, 1183

Suematsu, Y. 1990, Lect. Notes Phys., 367, 211

Torrence, C., \& Compo, G. P. 1998, Bull. Amer. Meteor. Soc., 79, 61

Tziotziou, K., Tsiropoula, G., \& Mein, P. 2004, A\&A, 423, 1133

Tziotziou, K., Tsiropoula, G., \& Sütterlin, P. 2005, A\&A, 444, 265 